\title{
Acute Leukemia in Children
}

\author{
Amanda Jensen Einungbrekke and Lukàš Plank \\ Jessenius Faculty of Medicine Faculty of Medicine in Martin, Comenius University in Bratislava, Martin 03601, Slovakia
}

\begin{abstract}
Acute leukemia is the most common childhood cancer and accounts for $31 \%$ of all cancers in children. There are two main types of acute leukemia. The most common is ALL (acute lymphoblastic leukemia) affecting the lymphoid lineage, and the more rare AML (acute myeloid leukemia) affecting the myeloid linage. The intention of this thesis is to follow the course of treatment from the admission to the hospital until the last check up and also see how a child will react to the treatment and side effects in later life. We studied literature and my own case records from the period when I was treated for ALL. From the literature and my case records, we can see that children tolerate treatment quite well. Due to rapid diagnostics and the possibility to give high doses chemotherapy, the overall prognosis appears to be very good. Today, acute leukemias of paediatric patients have a really favourable prognosis. The overall survival rate for ALL is higher than $80 \%$ and for AML $65 \%$. So the results are good, but there is still a long way to go before we can be satisfied. To date we do not have a contingency program for children treated for acute leukemia after 18 years of age (neither in Norway or Slovakia) so perhaps this should be a focus point in the future. It could be extended to follow up patients in adulthood in order to monitor late effects that may occur in later life after many years of treatment.
\end{abstract}

Key words: Acute leukemia, ALL, AML.

$\begin{array}{ll}\text { Abbreviations } \\ \text { ACTH } & \text { Adrenocorticotropic hormone } \\ \text { ALAT } & \text { Alanine aminotransferase } \\ \text { ALL } & \text { Acute lymphoblastic leukemia } \\ \text { AML } & \text { Acute myeloid leukemia } \\ \text { ASAT } & \text { Aspartate aminotransferase } \\ \text { BBB } & \text { Blood brain barrier } \\ \text { BM } & \text { Bone marrow } \\ \text { BNP } & \text { Brain type natriuretic peptide } \\ \text { CMV } & \text { Cytomegolavirus } \\ \text { CNS } & \text { Central nervous system } \\ \text { CRP } & \text { C-reactive protein } \\ \text { CT } & \text { Computertomography } \\ \text { CVC } & \text { Central vein catheter } \\ \text { FAB } & \text { French American British } \\ \text { FCA } & \text { Flow cytometric analysis } \\ \text { FISH } & \text { Fluorescence in situ hybridisation } \\ \text { Gamma GT } & \text { Gamma glutamyl transpeptidase } \\ \text { GIT } & \text { Gastrointestinal tract } \\ \text { GP } & \text { General practitioner } \\ \text { GFR } & \text { Glomerular filtration rate } \\ \text { GVHD } & \text { Graft versus host disease } \\ \text { Hb } & \text { Hemoglobin } \\ \text { Hct } & \text { Hematocrit } \\ \text { HE } & \text { Haematoxylin and eosin stain } \\ \end{array}$

Corresponding author: Amanda Jensen Einungbrekke, M.D., research fields: pathology, pediatrics and oncology.

$\begin{array}{ll}\text { HR } & \text { High risk group } \\ \text { HSV } & \text { Herpes simplex virus } \\ \text { IgA } & \text { Immunoglobulin A } \\ \text { IgG } & \text { Immuoglobulin G } \\ \text { IgM } & \text { Immunoglobulin M } \\ \text { IR } & \text { Intermediate risk group } \\ \text { LD } & \text { Lactate dehydrogenase } \\ \text { MRD } & \text { Minimal residual disease } \\ \text { MTX } & \text { Methotrexate } \\ \text { PCR } & \text { Polymerase chain reaction } \\ \text { PTSD } & \text { post traumatic stress disorder } \\ \text { RBC } & \text { Red blood cell count } \\ \text { SR } & \text { Standard Risk group } \\ \text { TBC } & \text { Thrombocytes } \\ \text { VZV } & \text { Varicella zoster virus } \\ \text { WBC } & \text { White blood cell } \\ \text { WHO } & \text { World Health Organization } \\ \text { NOPHO } & \text { Nordic Society of Paediatric Hematology and } \\ \end{array}$

\section{Introduction}

\subsection{History}

Acute leukemia, the most common form of paediatric cancer has become one of the success stories in the field of oncology. This once fatal disease is now curable in the majority of paediatric patients who 
receive fast and appropriate treatment $[1,2]$. In the 1970 era, approximately $10 \%$ of all children diagnosed with any type of paediatric cancer survived. Since then, there has been a tremendous development, and today $80 \%$ survive [3].

\subsection{Definition}

Acute leukemia is a malignant clonal disorder in one or more cell-lines in the hematopoietic system. It is the most common form of childhood leukemia, and comprises $1 / 3$ of all paediatric cancers (Fig. 1). There are two main types of acute leukemia found in children. The most common is ALL (acute lymphoblastic leukemia) (Fig. 2), and the more rare AML (acute myeloid leukemia). In Norway, there are 30 40 cases per year in children from $0 \sim 14$ years $[1,4]$.

ALL is a malignant clonal disorder that affects the lymphoid line in the hematopoietic blood system. It is a high count of immature lymphocytes, called lymphoblasts. The diagnosis can be confirmed when there are more than $25 \%$ lymphoblasts in the bone marrow picture.

The cancer cells can arrive either from B or T cells, where immature precursors of $\mathrm{B}$ are most common (90\%). In Norway and Slovakia, they follow NOPHO treatment protocol and differentiate ALL into three groups: standard risk, intermediate risk and high risk. Allocating each child to a risk group is dependent on cell type, leukocyte count, cytogenetics and how he/she responds to the given treatment $[1,4]$.

AML is a malignant clonal disorder of myeloid cells. It is a heterogeneous, clonal disturbance in the hematopoietic cells. They loose their capability to differentiate normally and to respond to normal regulatory mechanisms in proliferation. According to $\mathrm{FAB}$ classification, there must be a presence of at least $30 \%$ blasts in the BM. WHO classification has lowered the criteria to at least $20 \%$. AML accounts for approximately $15 \%$ of leukemias in children, and is rare. It can affect all age groups, and is more common in adults. Paediatric AML is more difficult to treat than ALL. And in comparison to ALL where it is more common to use WHO classification, we use FAB classification in AML. We have eight groups M0-M7. M3, also called "acute promyelocytic leukemia", has a special translocation $\mathrm{t}(15 ; 17)$. This type of leukemia is treated with a special protocol. Also M7 called "megakaryocytic leukemia" is extremely rare and very difficult to treat $[1,4,5]$.

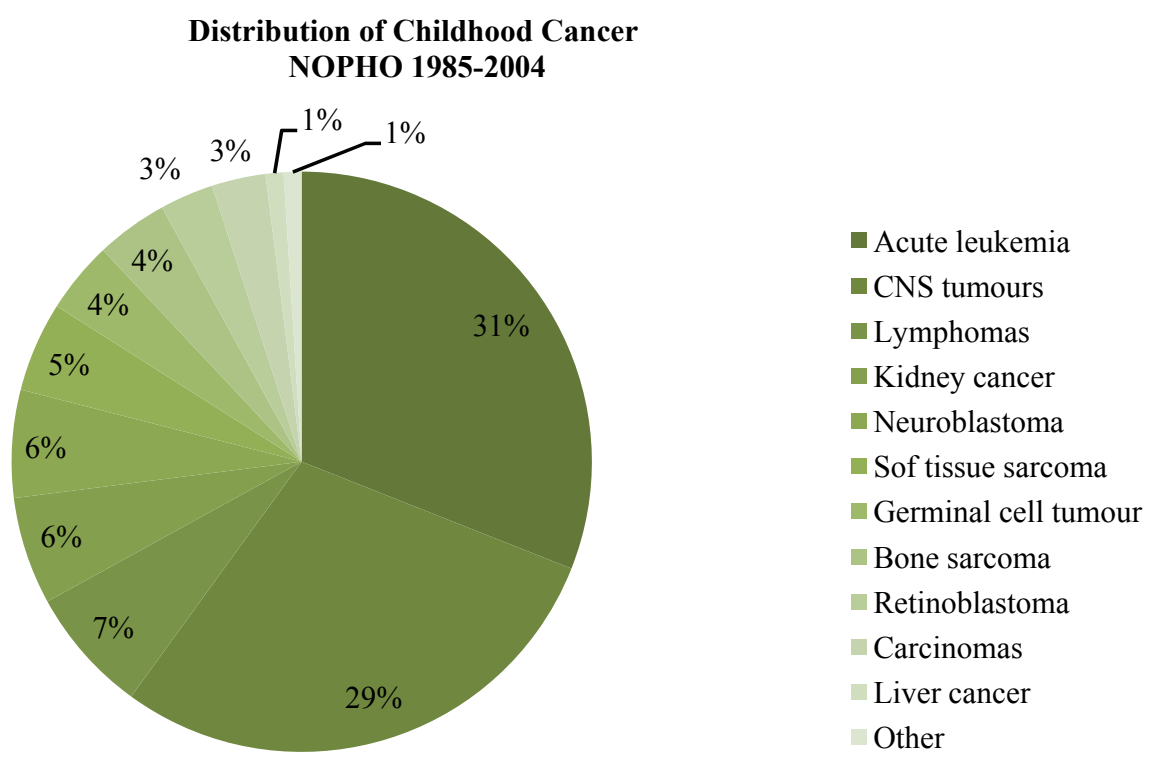

Fig. 1 Distribution of paediatric cancer [1]. 


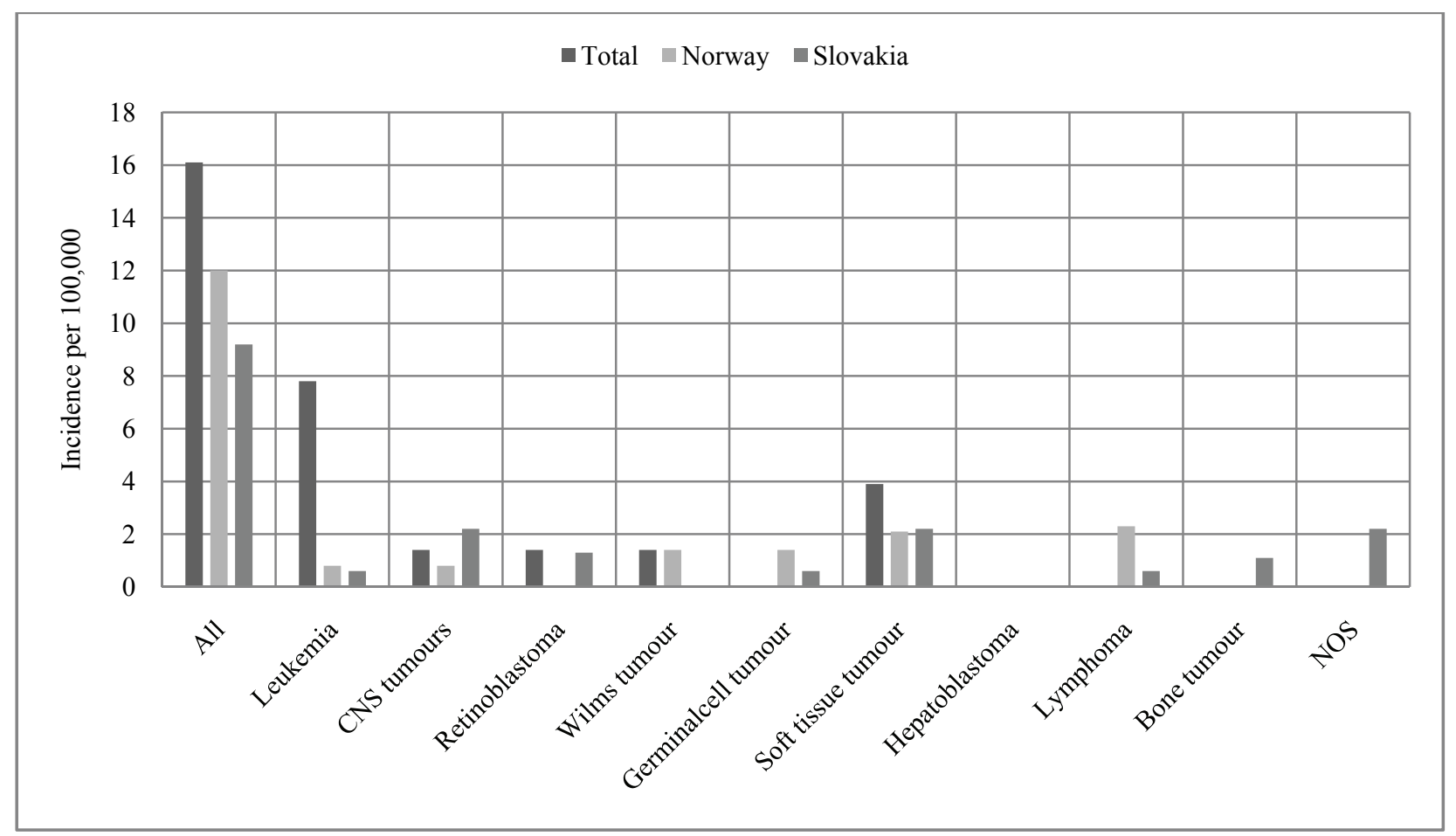

Fig. 2 Age specific incidence according to Norway and Slovakia.

\section{Theoretical Part}

\subsection{Classification}

Classification is the language of medicine: Diseases must be described, defined and named before they can be diagnosed, treated and studied, from WHO.

There are two classifications used in the definition of acute leukemias: FAB (French American British classidfication) and WHO (World Health Organisation). The difference between the two classifications is mostly related to the number of blasts in the BM. According the FAB criteria, number of blasts in the bone marrow should be more than $30 \%$. WHO has a criterion of $20 \%$. FAB classification is mostly based on morohology and cytochemistry. WHO on the other hand is based on clinical, cytogenetic and molecular abnormalities. WHO is the newest classification, and is widely used for leukemia, especially ALL.

\subsection{WHO Classification}

In 2007, WHO updated their classification of tumours of haematopoietic and lymphoid tissues [6].
This is a widely-used classification which can be found on many publications, such as the articles from Vardiman et al. [7] and Mautes et al. [8].

- AML and related precursor neoplasms;

- AML with recurrent genetic abnormalities:

(1) AML with $\mathrm{t}(8 ; \quad 21) \quad(\mathrm{q} 22 ; \quad \mathrm{q} 22)$; RUNX1-RUNX1T1;

(2) AML with inv (16) (p13.1; q22); CBFB-MYH11;

(3) Acute promyelocytic leukemia with $\mathrm{t}(15 ; 17)$ (q22; q12); PML-RARA;

(4) AML with $\mathrm{t}(9 ; 11)$ (p22; q23); MLLT3-MLL;

(5) AML with t(6;9) (p23; q34); DEK-NUP214;

(6) AML with inv (3) (q21; q26.2) or t(3;3) (q21; q26.2); RPN1-EVI1;

(7) AML (megakaryoblastic) with $\mathrm{t}(1 ; 22)$ (p13; q13); RBM15-MKL1;

(8) AML with mutated NPM1;

(9) AML with mutated CEBPA;

- AML with myelodysplasia-related changes;

- Therapy related myeloid neoplasms;

- Acute myeloid leukemia, NOS:

(1) AML with minimal differentiation; 
(2) AML without maturation;

(3) Acute myelomonocytic leukemia;

(4) Acute monoblastic and monocytic leukemia;

(5) Acute erythroid leukemia;

(6) Acute megakaryoblastic leukemia;

(7) Acute basophilic leukemia;

(8) Acute panmyelosis with myelofibrosis;

- Myeloid sarcoma;

- Myeloid proliferations related to down syndrome:

(1) Transient abnormal myelopoiesis;

(2) Myeloid leukemia associated with Down syndrome;

- Blastic plasmacytoid dendritic cell neoplasm;

- Acute leukemias of ambiguous lineage:

(1) Acute undifferentiated leukemia;

(2) Mixed phenotype acute leukemia with $\mathrm{t}(9 ; 22)$ (q34; q11.2); BCR-ABL1;

(3) Mixed phenotype acute leukemia with $\mathrm{t}(\mathrm{v} 11 \mathrm{q} 23)$; MLL rearranged;

(4) Mixed phenotype acute leukemia, B/myeloid, NOS;

(5) Mixed phenotype acute leukemia, T/myeloid, NOS;

- Precursor lymphoid neoplasms;

- B lymphoblastic leukemia/lymphoma:

(1) B lymphoblastic leukemia/lymphoma, NOS;

(2) B lymphoblastic leukemia/lymphoma with recurrent genetic abnormalities;

(3) B lymphoblastic leukemia/lymphoma with $t(9$; 22) (q34; q11.2); BCR-ABL1;

(4) B lymphoblastic leukemia/lymphoma with $\mathrm{t}(\mathrm{v} 11 \mathrm{q} 23)$; MLL rearranged;

(5) B lymphoblastic leukemia/lymphoma with $\mathrm{t}(12$; 21) (p13; q22); TEL-AML1 (ETV6- RUNX1);

(6) B lymphoblastic leukemia/lymphoma with hyperdiplodiploidy;

(7) B lymphoblastic leukemia/lymphoma with hypodiploidy (hypodiploid A);

(8) B lymphoblastic leukemia/lymphoma with $\mathrm{t}(5$; 14) (q31; q32); IL3-IGH;

(9) B lymphoblastic leukemia/lymphoma with $\mathrm{t}(1$;
19) (q23; p13.3); E2A-PBX1 (TCF3- PBX1).

\subsection{FAB Classification}

French American British classification is a much older classification, but still widely used today [9]. It is a much easier classification and mostly used in the diagnosis of AML.

FAB classification divide AML into eight subtypes M0-M7:

- M0-Minimally differentiated AML;

- M1-AML without maturation;

- M2-AML with maturation;

- M3-Acute promyelocytic leukemia;

- M4-Acute myelomonocytic leukemia;

- M5-Acute monocytic leukemia;

- M6-Acute erythroleukemia;

- M7-Acute megakaryocytic leukemia.

FAB classification divide ALL into three subtypes:

- L1-Childhood-ALL (B-ALL, and T-ALL);

- L2-Adult ALL (mostly T-ALL);

- L3-Burkitt type ALL (B-ALL).

\subsection{Feature AML and $A L L$}

Both AML and ALL have many of the same clinical features, and it is not possible to distinguish them only on clinical signs [10]. The importance of morphology, cytogenetic and immunophenotyping is invaluable when talking about diagnosis of acute leukemia. Table 1 shows the most contrasting features of AML and ALL (taken directly from Ref. [10]).

\subsection{Etiology}

There is no exact etiology which positively defines leukemia. To date, it would seem that acute leukemia is not related to any specific group of children and their respective circumstances and why they get the disease but some studies show that certain risk factors can precipitate it. The early detection and treatment is therefore more difficult. Twins have a higher frequency of occurrence if one of them develops leukemia. High doses of radiation in utero, so limiting the use of 
Table 1 Overview of the contrasting feature of AML and ALL [10].

\begin{tabular}{|c|c|c|}
\hline Feature & AML & ALL \\
\hline Common age & $\begin{array}{l}\text { Adults between } 15 \sim 40 \text { years; } \\
\text { Comprise } 20 \% \text { of childhood leukemia Children }<15 \\
\text { years }\end{array}$ & Comprise $80 \%$ of childhood leukemias \\
\hline Physical findings & $\begin{array}{l}\text { Splenomegaly }+ \text { Hepatomegaly }+ \text { Lymphadenopathy } \\
+ \text { Bony tenderness }+ \text { Gum hypertrophy }+\end{array}$ & $\begin{array}{l}\text { Splenomegaly ++ Hepatomegaly }++ \\
\text { Lymphadenopathy ++ Bony tenderness ++ CNS } \\
\text { involvement }++\end{array}$ \\
\hline Laboratory findings & $\begin{array}{l}\text { Low to high TLC; predominance of myeloblasts and } \\
\text { promyelocytes in blood and bone marrow; } \\
\text { Thrombocytopenia moderate to severe }\end{array}$ & $\begin{array}{l}\text { Low-to-high TLC, predominance of lymphoblasts in } \\
\text { blood and bone marrow; } \\
\text { Thrombocytopenia moderate to severe }\end{array}$ \\
\hline Diagnostic criteria & FAB types M0-M7 WHO criteria $\geq 20 \%$ blasts & $\begin{array}{l}\text { FAB types L1-L3, WHO types Pre B }(90 \%) \text { Pre T } \\
(10 \%) \text { WHO criteria } \geq 20 \% \text { blasts }\end{array}$ \\
\hline Cytochemical stains & $\begin{array}{l}\text { Myeloperoxidase }+ \text {, Sudan black }+ \text {, NSE }+ \text { in M4 and } \\
\text { M5, acid phosphatase (diffuse) }+ \text { in M4 and M5 }\end{array}$ & PAS + , acid phosphatase (focal) + \\
\hline Specific therapy & $\begin{array}{l}\text { Cytosine arabinoside, anthracyclines (daunorubicin, } \\
\text { adriamycin) and 6-thioguanine }\end{array}$ & $\begin{array}{l}\text { Vincristine, prednisolone, anthracyclines and } \\
\text { L-asparginase }\end{array}$ \\
\hline Immunophenotyping & CD13, 33, 41, 42 & $\begin{array}{l}\text { Both B and T cell AL Tdt +ve } \\
\text { Pre B: CD19,20 } \\
\text { Pre T: CD1,2,3,5,7 }\end{array}$ \\
\hline Cytogenetics & M3: $\mathrm{t}(15 ; 17) \mathrm{M} 4:$ in $(16)$ & Pre B: $\mathrm{t}(9 ; 21)$ \\
\hline Response to therapy & Remission rate low, duration of remission shorter & Remission rate high, duration of remission prolonged \\
\hline Median survival & 12 18 months & $\begin{array}{l}\text { Children without CNS prophylaxis } 33 \text { months, with } \\
\text { CNS prophylaxis } 60 \text { months; } \\
\text { Adults } 12 \sim 18 \text { months }\end{array}$ \\
\hline
\end{tabular}

diagnostic (CT) and therapeutic radiation are important initiatives. Some hereditary diseases or syndromes are more prone: Down's syndrome, Fanconi's anemia and ataxia telangiectasia has a slighter increased risk for developing acute leukemia $[1,4,11]$.

\subsection{Symptoms}

Symptoms of leukemia in children can vary greatly, and most of them are unspecific but can be a result of reduced bone marrow function. Other symptoms include bleeding, often present as petechiae is a feature of thrombocytopenia: Fever and infections (caused by low white blood cell count), some features of anemia such as fatigue, paleness and dyspnea. The child can also experience pain due to expansion of the bone marrow, often related to a joint or skeleton. The immature blasts can infiltrate other tissue and the patient will have symptoms like swollen lymph nodes, hepatomegaly, splenomegaly, swelling of testicles and kidney infiltration. If the CNS is involved, the child can complain about headache, double vision and pathological changes in cranial nerves. Usually the symptoms are vague, and the child can experience several infections before the diagnosis is confirmed $[1,4,12,13]$.

\subsection{Risk Groups}

Acute leukemia is divided into risk groups instead of stages as most other cancer types in adults.

According to NOPHO protocol, ALL is differentiated into three risk groups, based on cell type, leukocyte count at diagnosis, cytogenic criteria and treatment response:

(1) Standard risk:

- Age 1 17.9 years;

- Leukocytes at diagnosis $<100$;

- Pre-B ALL;

- No high-risk cytogenetic criteria;

- Positive response to therapy;

- MRD (minimal residual disease) $<10-3$ on Day 29 and Day 79 of therap;

(2) Intermediate risk:

- Age 1-17.9 years;

- Leukocytes at diagnosis $<100$;

- Pre-B ALL;

- CNS involvement (CNS3) at diagnosis; 
- Following cytogenetic changes: $t(1 ; 19)$ ic21amp dic 9; 20;

- No MRD marker available; or MRD > 10-3 Day 29 and $<10-3$ Day 79;

High risk group at the start (leukocytes $>100$ and/or $\mathrm{T}$ cell ALL) and no high risk cytogenetic changes (11q23 aberrations, hypodiploidy), but good response, MRD $<10-3$ day 29 and day 79.

(3) High risk:

- Age 1-17.9 years;

- Leukocytes at diagnosis $>100$ and/or T-ALL and $\mathrm{MRD}<10-3$ day 29. Or leuko- cytes at diagnosis $<100$ and pre-B ALL, but high risk cytogenetic changes (hypodiploidy, 11q23 aberrations);

- A poor treatment response, either MRD Day $29>$ 5 [4].

In addition, there are two special groups: infant leukemia and Philadelphia chromosome positive ALL. Both groups have their own protocol [4].

AML is not divided into risk groups the same way as ALL. It is divided into two risk groups based on the treatment response. Poor treatment response leads to upgrading to a higher risk group [4].

\subsection{Differential Diagnosis}

During the investigation of a child with suspected acute leukemia, it is important to consider other diagnosis as well. The differential diagnosis can be aplastic anemia, ITP (idiopathic thrombocytopenic purpura), acute anemia, Hemolytic uremic syndrome, infections with bone marrow suppression (CMV, EBV, parvovirus B19, HHV-6), rheumatoid arthritis, TMD (transient erythroblastopenia, myeloproliferative disease), DIC (disseminated intravascular coagulation) $[6,14]$.

\subsection{Prognosis}

With improved diagnostic, chemotherapy, hematopoietic stem cell transplantation and supportive care, the prognosis of paediatric acute leukemia has improved considerably during the past decades. The overall survival rate for ALL is $>80 \%$ and for AML 65\%. This development has been a fantastic success as 50 years ago success like this was simply the stuff of dreams. Today, the focus has changed somewhat and paediatric protocols have produce great results as research has shown that, by adapting treatment and using therapy that neither over treats nor under treats the patient, results are very much improved [15].

Even though we often refer to paediatric cancer a success story, some still do not survive. According to the paediatric cancer society in Norway (barnekreftforeningen.no) $4 / 5$ kids survive. So $20 \%$ of all paediatric patients treated for any type of cancer will die.

\subsection{Diagnosis}

Diagnosis is based on a combination of morphology, immunophenotyping, cytogenetic and other clinical features. AML and ALL have many of the same clinical features, and it is difficult to distinguish between them based solely on clinical manifestations.

2.10.1 ALL

Patients with ALL will also have symptoms of BM failure: thrombocytopenia, anaemia and neutropenia (Figs. 3 and 4). The leucocyte count can vary from leucopoenia to leucocytosis. The differential leucocyte count will show an increased number of lymphoblast. Other signs can be lymphadenopathy, hepatomegaly, splenomegaly, CNS infiltration, testicular enlargement and infections.

The morphology of the bone marrow will show lymphoblasts of either $\mathrm{B}$ or $\mathrm{T}$ cell lineage, reduced amount of red blood cells, and either absent or reduced number of megakaryocytes. The lymphoblasts in B-ALL often have scant cytoplasm and condensed nuclear chromatin. Periodic acid- Schiff, Acid phosphatase, Myeloperoxidase, Sudan Black and Non-specific esterase are used in characterisation of leukemic blasts.

The immunophenotyping is a fantastic method to 


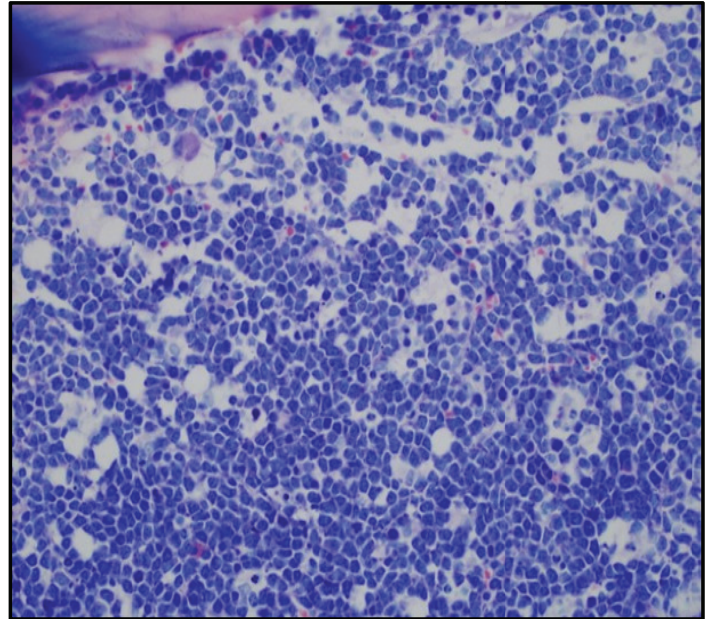

Fig. 3 BM, ALL, Giemsa stain and predominantly lymphoblasts. Photos from the register of Prof. Plank, Department of Pathology in Martin, Slovakia. Accessed April 2015.

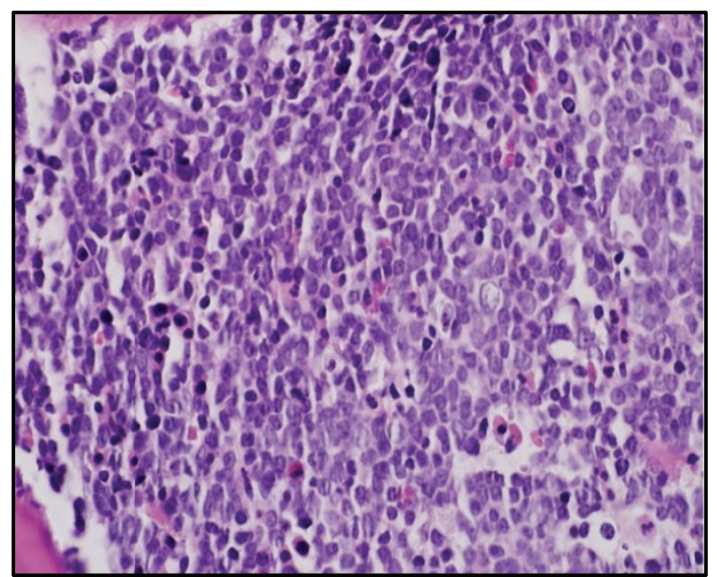

Fig. 4 BM, ALL, HE stain, predominantly lymphoblasts. Photos from the register of Prof. Plank, Department of Pathology in Martin, Slovakia. Accessed April 2015.

distinguish lymphoid from myeloid origin and vice versa. It will show which antigen and enzymes that are expressed on the cells. Tdt will always be present in pre-B and pre-T cell acute lymphoblastic leukemia. For B cell type, other markers typically present is CD19, CD10 and CD79a (Figs. 5 and 6). For T cell type it is commonly CD1, CD2, CD3, CD5 and CD7.

Cytogenetic abnormalities like translocations can have specific prognostic features. For example, pre-B ALL can show cytogenetic abnormality of $\mathrm{t}(9 ; 22)$, i.e., Philadelphia positive ALL. Leukemia with specific translocations often has specific protocols with individualized treatment.

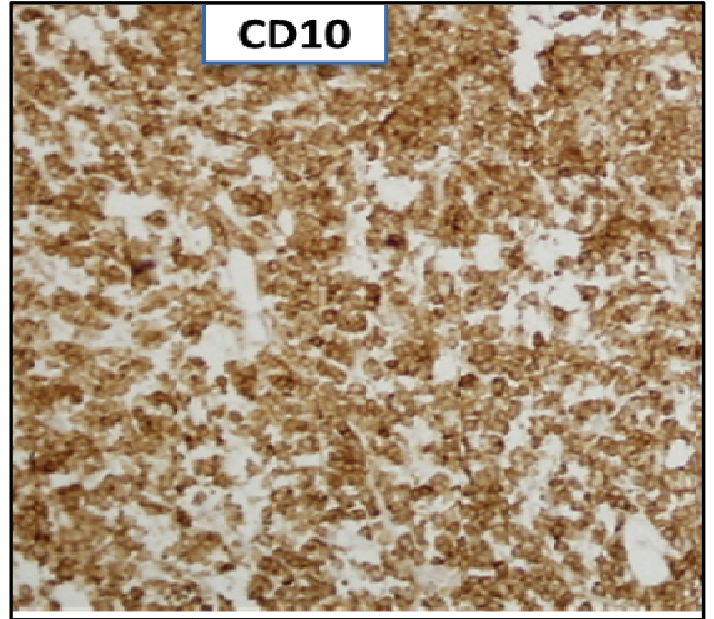

Fig. 5 CD10. Photos from the register of Prof. Plank, Department of Pathology in Martin, Slovakia. Accessed April 2015.

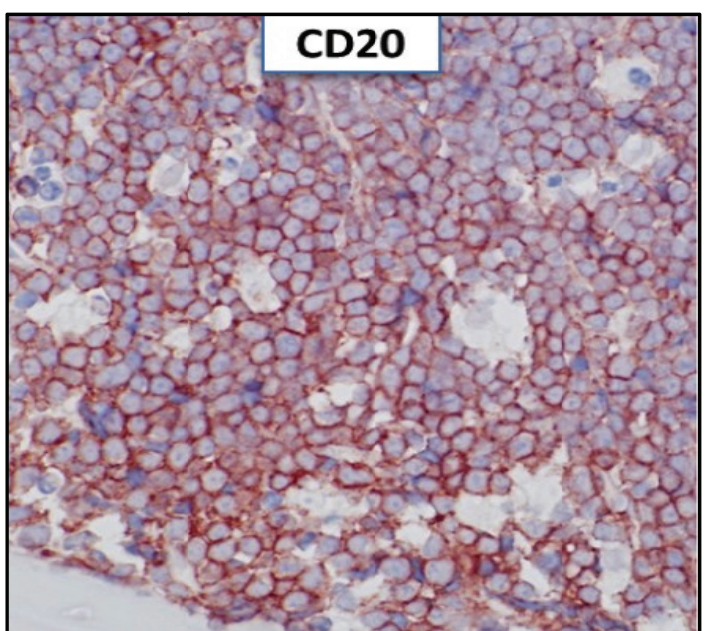

Fig. 6 CD20. Photos from the register of Prof. Plank, Department of Pathology in Martin, Slovakia. Accessed April 2015.

This is primarily a disease affecting children and $75 \%$ of the children are under six years of age. Approximately $85 \%$ of ALL is a pre B-cell type ALL. The $\mathrm{T}$ cell type often presents as a lymphoma with a mediastinal mass $[6,10,16]$.

2.10.2 AML

Patients who present with AML will also have symptoms of bone marrow failure; anaemia and thrombocytopenia. The WBC's can range from subnormal to highly elevated.

The morphology will show myeloblasts from either B or T cell origin, often with Auer Rods. Detection of leukemic cells is done by Romanowsky stain, 
sometimes in addition with Myeloperoxidase, Sudan Black, PAS (periodic acid-schiff), NSE (non-specific esterase) or acid phosphatase. The criterion for diagnosis of AML is $20 \%$ or more blasts in the BM (according to WHO).

The immunophenotyping will show leukemic expression of $\mathrm{CD} 13$ and $\mathrm{CD} 33$ antigens. M7 always show CD41 and CD42 positivity.

There are several different translocations, but the most consistent is probably M3 (15; 17) (q22; q22). This type has abnormal promyelocytes predominance and is associated with disseminated intravascular coagulation. AML M3 follows its own treatment protocol $[6,10]$.

\subsection{Treatment}

\subsubsection{ALL}

The treatment of ALL is chemotherapy. All paediatric patients in the Nordic countries receive the same treatment from the NOPHO ALL 2008 protocol. This protocol is again based on NOPHO ALL-92 and NOPHO ALL-2000. The treatment protocol lasts for two and a half year and is subdivided into: induction, consolidation and maintenance phase. A combination of vincristine, prednisolone, antracyclines, L-asparginase and methotrexate is used. The goal is of course to cure the patient, but there is balance between excessive toxicity and maintaining a high cure rate, neither overtreat nor undertreat. There is a variation in treatment dependent on the differing risk groups.

The induction phase lasts for six week (according to Einungbrekke Amanda Jensen's medical Journal, 1998 2009, founded by Hospital in Telemark and Oslo University Hospital) and the aim is to eradicate more than $99 \%$ of the blasts during this phase. In the induction phase, there is a combination of glucocorticoids (prednisone, prednisolone and dexamethasone), Vincristine, Antracyclines, Asparginase and Methotrexate. Some of the therapy is directed directly to CNS, often as intrathecal administration of methotrexate. On the other hand, dexamethasone has an indirect effect on CNS as it penetrates the BBB well. The patient can be considered for the consolidation phase once normal haematopoiesis is restored. The chemotherapy prescribed at this stage involves high dosage of Methotrexate, mercaptopurine, vincristine and high doses with asparginase. Allogenic stem cell transplantation can be a choice in very high-risk patients or those with poor response to treatment. Maintenance phase continues for two and half a half years. There is a combination of 6-mercaptopurin and methotrexate in tablet form, and also reinductions with vincristine/dexamethasone alternately with methotrexate. It exists own protocols for infant-ALL and Philadelphia-chromosome-positive ALL. As already stated, the future treatment of leukemia will focus more on reducing the toxic side effect of each component drug such as, for example, Doxorubicin [1, $4,10,15]$.

\subsubsection{AML}

For AML NOPHO-DBH-AML 2012-protcol is used. A combination of chemotherapy, antracyclines, cytarabin, etoposid is used. In AML, we divide into standard risk or high risk, according to how they respond to the first treatment. Patients in the high-risk group are candidates for stem cell transplantation in first remission. FAB M3 should be treated according to international APL protocol with all-trans-retinoic acid $[1,4,10,15]$.

\subsubsection{MRD}

Minimal residual disease is used to measure treatment response in patients, and to detect possible relapses. This is a newer method and can especially help patients with poorer prognosis. MRD is measured by flow cytometers and PCR [17].

Flow-cytometer is based on the detection of antigen expressed in the leukemic cells such as Tdt (Fig. 7) and CD10. There has been seen a correlation between MRD levels during clinical remission and treatment outcome. Acute leukemia is considered to be in remission when there is $<5 \%$ blasts in the BM. With early detection of relapse it is possible to change the 


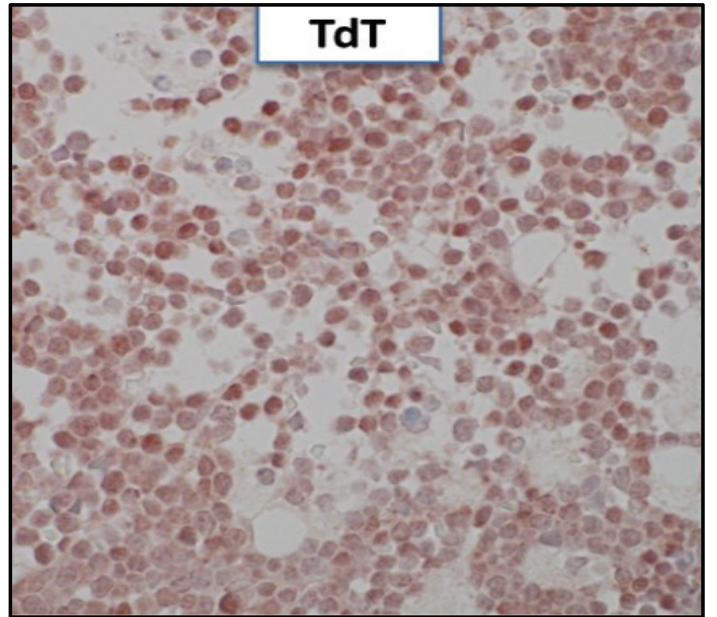

Fig. 7 Tdt. Photos from the register of Prof. Plank, Department of Pathology in Martin, Slovakia. Accessed April 2015.

treatment strategy, and possibly improve the cure rate $[1,4,6,18]$.

\subsubsection{Stem Cell Transplantation}

BM transplantation from a suitable allogeneic donor can cure an otherwise incurable leukemia. For ALL allogeneic hematopoietic stem cell transplantation should be care- fully selected. It is indicated in very high-risk patients and those with poor treatment response. Some subtypes of ALL, such as $\mathrm{PH}+$ ALL, have been shown to benefit from transplantation. HLA typing of the donor to find the most suitable match is very important for the result and to avoid GVHD. Allogeneic stem cell transplantation in AML patients is used more compared to ALL patients. In the Nordic countries, it is indicated for children with high-risk criteria in remission, and for second remission. Long-term complications develop more often in paediatric patients treated with Bone marrow transplantation compared to those receiving just chemotherapy. So this is of course something to take into consideration when considering the suitability of stem cell transplantation $[4,10,15,19]$.

\subsection{Side Effects during Treatment}

Adverse reactions of AML and ALL are quite similar, due to many of the same chemotherapeutic drugs. The treatment is very intense though and it is crucial with supportive therapy to help the child through this year with chemotherapy. Supportive therapy will also have a positive effect on side effects, the side effects will be reduced and thus lead to a better life during and after treatment.

Doxorubicin/adriamycin will very often give adverse reactions as leucopenia, neutropenia, anemia, thrombocytopenia, inflammations in GIT, diarrhea, nausea, vomiting, hair loss, infection, decreased appetite, decreased ejection fraction and congestive heart failure. The Norwegian Health care recommends regular check ups with echocardiography during and after treatment with Doxorubicin [20].

Vincristine often gives side effects as alopecia, muscle weakness, muscle atrophy, loss of tendon reflexes, leg pain, jaw pain, paresthesia neuritis pain and leucopenia [21].

Methotrexate will give leukopenia, neutropenia, thrombocytopenia, anemia, stomatitis, nausea, vomiting, infections, alopecia, decreased kidney function and fatigue. Calcium folinat (leukovorin) injection is used as an antagonist to prevent adverse reactions [22].

Prednisolone will give side effects in the form of Cushing symptoms, skin atrophy, decreased wound healing, hypokalemia, sodium retention, muscle atrophy, growth retardation. It will inhibit excretion of ACTH and cortisone [23].

Asparginase can give allergy, anaphylactic reaction, thromboembolic complications and hemorrhagic pancreatitis [24].

Cytarabin often gives infections, BM suppression in form of thrombocytopenia, anemia, megaloblastic anemia leucopenia and decreased number of reticulocytes. Other common adverse reactions are oral/anal infections or ulcerations, diarrhea, nausea, vomiting and itching $[4,24,25]$.

\subsection{Side Effects Later in Life and Follow Up Care}

Childhood cancers are different compared to cancer in adults. A child is under development when he/she 
gets the disease, and the cancer and chemotherapy can damage the maturation process. They have also longer life expectancy compared to adults and therefore more time for side effect to occur.

In recent past years, side effects in children have not been fully focused upon as understanding the main concern has been curing the patient. Fortunately, in most cases, this was successful. However, the intense treatment creates additional problems in later life with which the patient has to cope, sometimes for the rest of their lives. Currently, most children are monitored up to eighteen years of age but thereafter have to contact their GP should they become unwell. GP's are not cancer specialists and are therefore sometimes unaware of the many side effects resulting from different chemotherapeutic drugs that have been administered to the patient.

Overall childhood leukemia is quite rare, therefore it is quite difficult to find sufficient cases to study and many childhood cancer survivors are now in adult hood and devoid of any follow up consultations. Side effects after acute leukemia vary between individual risk groups and is consequently dose related. Most of the survivors will experience changes, some to a greater or lesser degree. There could be hormonal changes, the fertility can be reduced, Heart function can be decreased, fatigue, problems with the musculoskeletal system, concentration problems, psychological difficulties and secondary malignancy.

The fertility questions is a difficult one, both the disease itself and the treatment can give reduced fertility. The ovaries in girls are mostly at risk if the treatment is received at post-pubertal age.

Cardiotoxicity is recognised complication of doxorubicin treatment. The chemotherapy is believed to impair the myocardial growth in a dose related fashion. Patients treated for ALL will have an echocardiography examination on the last check up. An AML patient on the other hand will have echocardiography examinations during the whole treatment period. In the new guidelines from
Helsedirektoratet, they recommend all patients treated for acute leukaemia to go for regular check up with echocardiography also after treatment and in adult life.

Problems with musculoskeletal system may appear as growth disturbances, osteoporosis, secondary tumors and reduced bone density.

Secondary malignancy may be the survivors biggest fear. They have 3 10 times higher risk of developing malignancy compared to the rest of the population. The chemotherapy can damage the genetic material in the nucleus, and a new malignancy can develop.

Fatigue is a newer term in the cancer context. After treatment, the patient can experience chronic tiredness and a noticeable change in energy levels but this will vary from patient to patient [1].

\section{Practical Part}

\subsection{Case Record in November 1998}

A 7-year-old girl is presented to the ER with fever and abdominal pain. They suspect appendicitis. She has had abdominal pain on and off for the last two months, and complained about leg pain the last year. At the ER, she is pale and weak, but in an overall good condition. The vital parameters are normal. The only pathological findings are lymphadenitis at the neck and a systolic murmur Grade 2. The blood tests show CRP: 18, WBC: 2.0, Hb: 8.1, Tbc: 85 (mild pancytopenia). The assessment is that it seems viral characterized. She is admitted to the paediatric department.

Two days later, they take a new blood test: $\mathrm{Hb}: 7.2$, WBC: 2.0, Tbc: 90, CRP: 44, Reticulocytes: 0.3. The peripheral blood smear shows a low WBC's count, granulocytes $45 \%$, and a predominance of immature mononuclear cells. The same day in anaesthesia, a BM puncture from right crista shows a very monotonous picture of uniforms blasts, $90 \%$ nucleated.

Two days later, she is transferred to a regional hospital for further investigation and a new BM biopsy. This shows the same picture of monotonous lymphoblast. An immunological examination displays that all bone marrow cells emanate from pre B-cell 
stadium. She got the diagnosis acute lymphoblastic leukemia standard risk group.

\subsection{Diagnosis}

Fig. 8 shows BM from this case record.

\subsection{Immunophenotyping}

BM biopsy is dated November 11, 1998. This is the result from the immunophenotyping, a diagnostic method performed on cells from the BM (Table 2).

\subsubsection{Assessment of Bone Marrow Biopsy}

Seen in bone marrow is a nearly uniform population of small to medium sized cells with low side scatter.
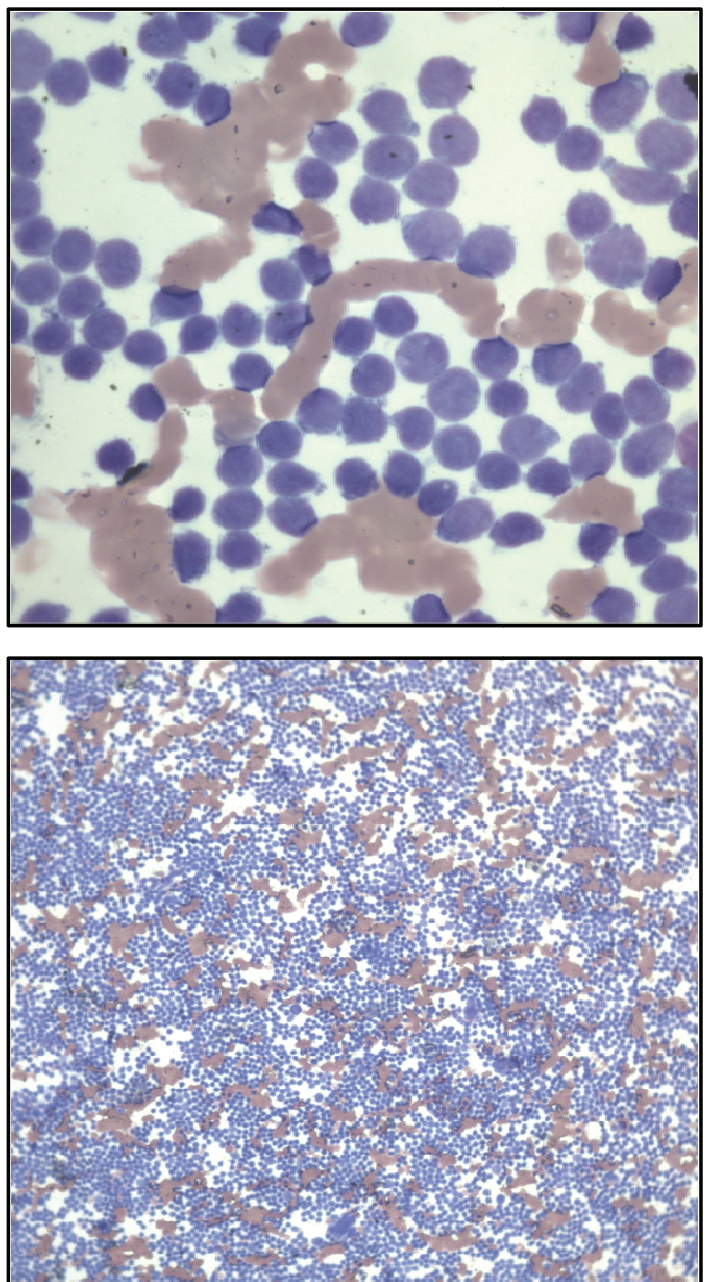

Fig. 8 BM from November 1998. Deadly monotomy, a tedious picture of small blue lymphoblasts, barely other cells visible. The red cells are compared clumping RBC's. Einungbrekke Amanda Jensen's Personal Medical Journal, 1998 2009, Hospital in Telemark and Oslo University Hospital.
Table 2 Antibodies against the BM.

\begin{tabular}{|c|c|}
\hline Items & Value \\
\hline \multicolumn{2}{|l|}{ B cell-line } \\
\hline CD10 (a-CALLA, 552/36) & 72 \\
\hline CD19 (HD37 PE) & 96 \\
\hline CD20 (B-Ly1) & 2 \\
\hline CD24 (CLB-134) & 96 \\
\hline \multicolumn{2}{|l|}{ CD37 (IGB1) } \\
\hline \multicolumn{2}{|l|}{ CD38 (OKT10) } \\
\hline \multicolumn{2}{|l|}{ T cell-line } \\
\hline CD7 (DK24) & 2 \\
\hline CD5 (UCHT-2) & 72 \\
\hline CD2 (MT910) & 12 \\
\hline \multicolumn{2}{|l|}{ CD1 (OKT 6) } \\
\hline CD3 (UCHT-1) & 2 \\
\hline \multicolumn{2}{|l|}{$\begin{array}{l}\text { CD4 (MT 310) } \\
\text { CD8 (DK25) }\end{array}$} \\
\hline \multicolumn{2}{|l|}{ TCR alpha/beta ( T-cell SCIENCES) } \\
\hline \multicolumn{2}{|l|}{ TCR g/d (T-cell diagn.) } \\
\hline \multicolumn{2}{|l|}{ Pan myeloid } \\
\hline CD13 (WM47) & 16 \\
\hline CD33 (WM 44) & 1 \\
\hline \multicolumn{2}{|l|}{ CD68 (EBM M) } \\
\hline \multicolumn{2}{|l|}{ Various cell types } \\
\hline \multicolumn{2}{|l|}{ HLA-ABC (E2-14) } \\
\hline HLA-DR (CR3/43) & 97 \\
\hline CD34 (HPCA-2) & 96 \\
\hline CD45 (LC, T29/33) & 91 \\
\hline \multicolumn{2}{|l|}{ CD56 (Leu 19) } \\
\hline \multicolumn{2}{|l|}{ CD103 (HML-1) } \\
\hline Anti-kappa & 2 \\
\hline \multicolumn{2}{|l|}{ Anti-lambda } \\
\hline \multicolumn{2}{|l|}{5 a-desmin } \\
\hline \multicolumn{2}{|l|}{ Cytokeratin (AE1/AE3, ICN) } \\
\hline \multicolumn{2}{|l|}{ Monocyte line } \\
\hline CD14 (TUK4) & 1 \\
\hline \multicolumn{2}{|l|}{ CD11b (44) } \\
\hline IgG1 & 1 \\
\hline \multicolumn{2}{|l|}{ Platelets and megakaryocytes-line } \\
\hline \multicolumn{2}{|l|}{ CD41 (ITI-PL2) } \\
\hline \multicolumn{2}{|l|}{ CD42 (ITI-PL1) } \\
\hline CD42 (ITI-PL1) & $<1$ \\
\hline \multicolumn{2}{|l|}{ Erythroid line } \\
\hline Glycophorin A (1C10) & 3 \\
\hline Antigens close to stem cells & \\
\hline CD34 (HPCA 2) & \\
\hline Tdt (aTdt) & \\
\hline Activation antigens & \\
\hline CD25 (A-Tac, ACT-1, CD25) & \\
\hline CD30 (BER-H2) & \\
\hline CD71 (T58-1) & \\
\hline Granulocyte line & \\
\hline CD15 (C3D-1) & 1 \\
\hline
\end{tabular}


The cells are positive for CD45 and the B-cells markers CD19, CD24 and HLA-DR.

CD20 and surface immunoglobulin are negative. Stem cell-associated CD34 is positive on all cells. $\mathrm{CD} 10$ and $\mathrm{CD} 2$ are expressed respectively on $77 \%$ and $76 \%$ of the cells Absence of myeloid antigens CD13, CD14, CD15, CD33.

Diagnose: pre B-ALL according to FCA biphenotypic CD19, 10, 24, 34, 45, DR, 2; Standard risk group.

Comments to the immunophenotyping:

As we can see from the diagnosis, they called it a biphenotypic leukemia. This is what we today call aberrant expression of markers from another cell line. So the diagnosis would today most likely be: Pre-B ALL with aberrant expression of CD2 (usually a T-cell maker) but can also be expressed on leukemic $\mathrm{B}$ cells. And from the immunophenotyping CD3 and CD7 is both negative, and excludes a possible biphenotypical leukaemia. It is important to remember that this is more than 15 years ago, and the criteria for biphenotypic leukemia have been changed and tightened. It is not enough that the leukemic cells express just one marker from another cell line today.

Leukemia of ambigious lineage means there is no clear evidence of differentiation along a single lineage (WHO). This type of leukemia is very rare and account for $<4 \%$ of all cases. This group of leukemia is today divided into acute undifferentiated leukaemia and Mixed phenotype acute leukemia. Cases that in the past were diagnosed as undifferentiated leukemia may today be diagnosed as leukemia of unusual lineages. And cases that have been reported as Biphenotypic leukemia may today be a clear lymphoid or myeloid leukemia. The reason for this is that the classification has changed the last two decades; today it is based on the clinical features, the immunophenotyping, the morphology and the cytogenetic. And it exists several other criteria before we can call it a mixed phenotype acute leukemia [6].

Tdt is almost always expressed in acute leukemia (even though there exist some exceptions). Tdt is not mentioned in this immunophenotyping, but it probably was expressed. CD34 was neither mentioned in the immunophenotyping, but mentioned in the result. So they probably forgot to mention Tdt.

\subsubsection{Analysis}

When a patient is diagnosed with acute leukemia or there is a suspicion for the disease, there is a standard procedure to check for some immunological abnormalities, do a microbiological examination and a cytological examination:

12.11 .1998

$\operatorname{IgG} 13,8 \mathrm{~g} / 1(4,3-13,6) \operatorname{IgA} 1,07 \mathrm{~g} / 1(0,35-3,00) \operatorname{IgM}$ $1,77 \mathrm{~g} / 1(0,20-1,70)$

Microbiological examination:

11.11.1998

Anti-CMV: Negative Anti-HSV IgG: NEGATIVE Anti.measelsvirus IgG: Weak positive Anti-VZV IgG: Positive

Cytological examination

19.11.1998

Karyotype:

46, XX

FISH examination

03.12.1998

FISH examination for 12; 21 translocation: Negative

\subsection{Treatment}

The first day after the diagnosis was set; She got a central venous catheter. The chemotherapy regimen could start already the day after the CVC in-plant. So six days after the admission to ER, chemotherapy after Nordic protocol could start; see theoretical part or NOPHO for the whole protocol. During the treatment period, she underwent regularly blood tests.

After the treatment protocol which lasts two and a half year, regular check-up is started. During the 1st year, there is control each month, 2nd year every 2nd month, 3rd year every 3rd month, 4th year every 4th month, 5th year every 4 th or 5 th month, 6th year ever 6th month, and after this control each year until 18 years of age. 
At her last control as an 18-year-old girl, routine cardiac examination after treatment of ALL Echocardiography shows a structural and functionally normal heart. Left ventricle measure diastolic 54.4, set score 0.2 and shortening fraction $30 \mathrm{~A}$ normal heart at that time.

\subsection{Adverse Reactions during Treatment}

During the treatment period with chemotherapy, she experienced a lot of side effects. The chemotherapy given is really toxic, and will for sure give some adverse reactions. Not all will experience the same problems, but all will more or less have side effects.

Some of the chemotherapy made her really sick, and especially nausea was a problem. It usually stopped 1 2 days after each cure, and then it start again when a new cure began. Mucositis was another, it occurred frequently when the blood values was low. This led to some nutrition problems.

Vincristine gave her neuromuscular manifestations with sensory and motor disturbances in her legs. She often waked up during the night with big leg pain.

Changed appearance was maybe the worst side effect at that moment. Even though it really does not matter, losing her hair was a big breaker. The steroids gave her typically moon face and an enormous appetite, She could wake up in the middle of the night so enormously hungry. Another side effect of steroids is mood swings, not only affecting the patient but her family as well.

There are infections, due to low WBC's count. So she had to be isolated from the community in some periods. It was strictly forbidden to go to shops, cinema or school.

\section{Discussion}

Maybe the worst part of being a cancer kid is the life after treatment. When you have to deal with the fact that you once had a life threatening illness, survived and are expected to live a full and happy life thereafter. The truth is however that your every waking moment nurtures questions such as why me, will my life be normal again, but the darkest thoughts are those about possible secondary malignancy.

I believe we all have a choice about how to deal with life after a really though and devastating period and the option vary for each and every individual. I actually do not remember how I was told or even how I reacted, probably because I was only seven years old at the time. From the journals I read I did not want to know anything about chemo and treatment at that particular point. But even when you are sick, life for us all goes on and we have to deal with whatever cards you are dealt and believe there will be light at the end of the dark tunnel. And there was a light because 15.05.2001 was my last chemo session. After more than two years in hospital, regular check ups started. Blood tests, talking with doctors, how do you feel etc. was the regular conversation with my doctor. I believe many cancer sufferers feel different after their experience and I know I did but maybe that was because I was so young and face with such a serious diagnosis. When talking about my future with the doctors I always insisted that I would never go to a hospital voluntarily again because I just wanted to forget and live life like my friends, no blood test and medical discussions. As I grew older, I realized that I never will forget, and my cancer story will always be with me. It is not me, but it is a part who I have become.

I was lucky I got standard risk ALL with really good prognosis and few late damage effects compared to others. My ultimate dream is that one day it will be possible to help this patient group by being able to answer questions related to their childhood illness and talk to them about side effects and when they may appear. Many survivors do not know about the side effects that can appear. They have to be able to seek council from understanding and sympathetic professionals about their physical and/or psychological issues later in life. Their worries may not be cancer related but they are still their worries and need to be addressed as a connection could be present. For me, on 
a very personal level, there is a much bigger and wider perspective because who cares about possible side effects when you have been given a second chance at life itself. For that chance, I am eternally grateful.

Today, the overall survival rate for ALL is $>80 \%$ and for AML $65 \%$. The treatment is very effective and the results are good. Even with such good results there is a long way to go when we think about side effects later in life. A child has longer life expectancy compared to an adult and therefore more time for side effects to occur. Most of the survivors will experience problems, some to a greater or lesser degree. There could be psychological difficulties, hormonal changes, the fertility can be reduced, heart function can be decreased, fatigue, problems with the musculoskeletal system, concentration problems and the worst of them all secondary malignancy. As already stated, the focus has changed somewhat the last years due to more awareness on side effects. I think the focus in the coming years will be more related to possible side effects after treatment and to make as few complications as possible after chemotherapy treatment.

\section{Conclusions}

Topic of this thesis was acute leukemia in children. Through study of literature and my own case record of ALL, I gained knowledge about this disease. It made me more aware than ever, not only of the though treatment a child has to undergo, but also just how much progress has been made in the diagnosis and treatment of leukemia.

The aim of my diploma thesis was to focus primarily on side effects and follow up care after treatment. To date, we have come a long way in the treatment of paediatric leukemia as can be seen from the result statistics. Modern techniques enable the early and rapid diagnosis of the disease and with high doses of chemotherapy it is possible to cure almost all diagnosed acute leukemia. However, such high doses can result in side effects later in life and although we monitor patients up to the age of eighteen there is an urgent need for a special department where consultants, who are fully conversant with the disease and the subsequent side effects are available, to whom such people can go as opposed to a GP who may or may not be so knowledgeable. Let's hope it can be introduced a follow up program to prevent complications for all affected patients. This must be the next achievement in the field of paediatric oncology, and will definitively give this patient group a better life and less side effects.

\section{Acknowledgments}

I wish to acknowledge and thank everyone who has helped and supported during the time I was writing this thesis. My most sincere and grateful thanks to Prof. Lukàš Plank, M.D., Ph.D., who guided and supported me during the process and his wealth of experience and knowledge was inspiring and further motivated to progress further in my chosen field. My family have featured amazingly with their ever ready and unwavering support and I owe them deep gratitude and heartfelt thanks, especially my father who is a constant source of encouragement and motivates me continually towards my goals. I also want to thank my friends Ray and Beate for all support during this period. Many thanks to both Oslo University Hospital and the Hospital in Telemark who provided the relevant details from their medical records relating to my treatment in the period of 1998-2009.

\section{References}

[1] Helsedirektoratet. 2014. National Action Program with Guidelines for Diagnosis Treatment and Follow Up for Childhood Cancer. Technical Report. (in Norwegian)

[2] Greaves, M. 1993. "A Natural History for Pediatric Acute Leukemia.” Blood Journal 82 (4): 1043-51.

[3] The Norwegian Cancer Society. 2015. "Childhood Cancer Highly on the International Agenda." The Norwegian Cancer Society. Accessed February 10, 2016. https://kreftforeningen.no/aktuelt/siste-nyheter/barnekrefthoyt-pa-internasjonal-agenda/.

[4] Oncology Encyclopedia. 2014. "Diagnoses Leukemia." Oncolex. Accessed February 2, 2016. http://www.oncolex.org/en/Childhood-cancer/Diagnoses/ 
AcuteLeukemia.aspx.

[5] Ahmadi, S., Rezaei-Tavirani, M., Divsalar, A., Khodakarim, S., and Tahmasebi, L. 2012. "Study of Contributing Factors for Cure Response in Patients with Acute Myeloblastic Leukemia (AML)." Journal of Life Sciences 6: 570-6.

[6] Swerdllow, S., Campo, E., and Harris, N. L. 2008. WHO Classification of Tumours of Haematopoietic and Lymphoid Tissues. Lyon: IARC Press.

[7] Vardiman, J. W., Thiele, J., Arber, D. A., Brunning, R. D., Borowitz, M. J., Porwit, A., et al. 2009. "The 2008 Revision of the World Health Organization (WHO) Classification of Myeloid Neoplasms and Acute Leukemia: Rationale and Important Changes." Blood Journal 114: 937-51.

[8] Matutes, E., Pickl, W. F., Van’t Veer, M., Morilla, R., Swansbury, J., Strobl, H., et al. 2011. "Mixed-Phenotype Acute Leukemia: Clinical and Laboratory Features and Outcome in 100 Patients Defined According to the WHO 2008 Classification." Blood Journal 117: 3163-71.

[9] Lilleyman, J. S., Hann, I. M., Stevens, R. F., Eden, O. B., and Richards, S. M. 1986. "French American British (FAB) Morphological Classification of Childhood Lymphoblastic Leukaemia and Its Clinical Importance." Journal of Clinical Pathology 39: 998-1002.

[10] Mohan, H. 2010. Textbook of Pathology. Sixth Edition. New Delhi: Jaypee Brothers.

[11] Belson, M., Kingsley, B., and Holmes, A. 2007. "Risk Factors for Acute Leukemia in Children: A Review." Environmental Health Perspectives 115: 138-45.

[12] Jonsson, O. G., Sartain, P., Ducore, J. M., and Buchanan, G. R. 1990. "Bone Pain as an Initial Symptom of Childhood Acute Lymphoblastic Leukemia: Association with Nearly Normal Hematologic Indexes." The Journal of Pediatrics 117: 233-7.

[13] Kreftlex. 2016. "Symptoms of Leukemia." Institutt Kreftgenetikk og Informatikk. Accessed January 16, 2016. http://kreftlex.no/Leukemi/BAKGRUNN/Symptomer?Sea rchText=akutt\%20leukemi(Symptomer på leukemi).

[14] Carroll, W. L., Bhojwani, D., Min, D. J., Raetz, E., Relling, M., Davies, S., et al. 2003. "Pediatric Acute Lymphoblastic Leukemia." ASH Education Program Book
2003 (1): 102-31

[15] Pui, C., Schrappe, M., Ribeiro, R. C., Niemeyer, C. M. 2004. "Childhood and Adolescent Lymphoid and Myeloid Leukemia." ASH Education Program Book 2004 (1): 118-45.

[16] Casasnovas, R., Slimane, F. K., Garand, R., Faure, G. C., Campos, L., and Deneys, V. 2003. "Immunological Classification of Acute Myeloblastic Leukemias: Relevance to Patient Outcome." Leukemia 17: 515-27.

[17] Campana, D. 2012. "Minimal Residual Disease." Leukemia Supplements 1: 3-4.

[18] Pui, C. H., and Evans, W. E. 2006. "Treatment of Acute Lymphoblastic Leukemia." New England Journal of Medicine 354: 166-78.

[19] Appelbaum, F. 1997. "Allogeneic Hematopoietic Stem Cell Transplantation for Acute Leukemia." Seminars in Oncology 24 (1): 114-23.

[20] Pharmaceutical Catalogue for Norwegian Doctors. 2015. "Adriamycin." Felleskatalogen AS. Accessed December 22, 2015. http://www.felleskatalogen.no/medisin/adriamyc in-pfizer-545768.

[21] Pharmaceutical Catalogue for Norwegian Doctors. 2015. "Vincristine." Felleskatalogen AS. Accessed December 22, 2015. http://www.felleskatalogen.no/medisin/vincristine-p fizer-565216.

[22] Pharmaceutical Catalogue for Norwegian Doctors. 2015. "Methotrexate." Felleskatalogen AS. Accessed December 22, 2015. http://www.felleskatalogen.no/medisin/methotre xate-pfizer-578020.

[23] Pharmaceutical Catalogue for Norwegian Doctors. 2015. "Prednisolone." Felleskatalogen AS. Accessed December 22, 2015. http://www.felleskatalogen.no/medi sin/prednisolon-takeda-562951.

[24] Health Library for Norwegian Doctors. 2015. "Cytostatikabehandling." Helsebiblioteket. Accessed December 15, 2015. http://www.helsebiblioteket.no/retnin gslinjer/pediatri/hematologi-og-onkologi/cytostatikabehan dling.

[25] Pharmaceutical Catalogue for Norwegian Doctors. 2015. "Cytarabine." Felleskatalogen AS. Accessed December 22. http://www.felleskatalogen.no/medisin/cytarabine-pfizer-5 47759 . 